\title{
The CD271 expression could be alone for establisher phenotypic marker in Bone Marrow derived mesenchymal stem cells
}

\author{
Edgardo Flores-Torales ${ }^{1,2}$, Arturo Orozco-Barocio ${ }^{1}$, Oscar R. Gonzalez-Ramella ${ }^{2,3}$, \\ Antonio Carrasco-Yalan ${ }^{2}$, Karlen Gazarian ${ }^{4}$, Silvio Cuneo-Pareto ${ }^{2}$
}

${ }^{1}$ Departamento de Biología Celular y Molecular, Centro Universitario de Ciencias Biológicas y Agropecuarias, Universidad de Guadalajara, México

${ }^{2}$ Sangre de Cordón S.A. de C.V., Guadalajara, México.

${ }^{3}$ Departamento de Fisiología, Centro Universitario de Ciencias de la Salud, Universidad de Guadalajara, México

${ }^{4}$ Departamento de Biología Molecular y Biotecnología, Instituto de Investigaciones Biomédicas, Universidad Nacional Autónoma de México, México

\begin{abstract}
Mesenchymal stem cells (MSCs) are of great interest for their potential use in cellular therapies. To define the population more precisely, diverse surface markers have been used. We propose here to use CD271 as the sole marker for MSCs in fresh bone marrow. We compared CD271 ${ }^{+}$populations to the presence or absence of five defined markers for

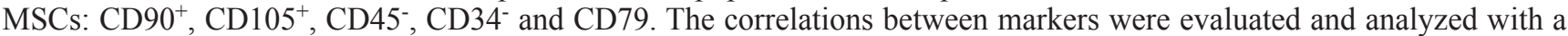
Pearson's correlation test. We found that the average percentage of cells expressing the combination of markers CD90+,

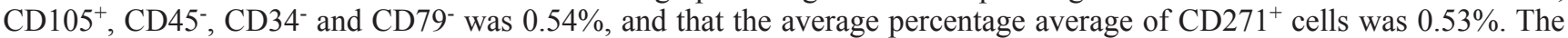
results were significant $(\mathrm{p}<0.05)$. The exclusive use of CD271 as a marker for MSCs from fresh samples of bone marrow appears to be highly selective. Using CD271 as the sole identification marker for MSCs could reduce costs and accelerate the process of identifying MSCs for the field of cellular therapy.
\end{abstract}

Key words: mesenchymal stem cells, CD271, bone marrow, surface markers.

\section{Introduction}

Mesenchymal stem cells (MSCs) reside in small numbers in bone marrow (BM) as well as other tissues and have been shown to be relatively easy to expand ex vivo $[1,2]$. SCs differentiate in vitro and in vivo into different connective tissue cells types including osteoblasts, adipocytes, chondrocytes, endothelial and myogenic cells [3-7]. This multidifferentiation potential of MSCs raised a clinical interest to use these cells for regeneration purposes e.g., in treating osteogenesis imperfecta, stroke or myocardial infarction. MSCs also lack MHC-II antigens and possess immunomodu-

Correspondence: Sangre de Cordón S.A. de C.V., Av. José M. Morelos 2006, Col. Ladrón de Guevara,

C.P. 44600, Guadalajara, Jalisco, México;

e-mail: edgardo.flores@sangredecordon.com latory properties, which enable them to treat acute graft-versus-host disease (GvHD) after hematopoietic transplantation [8-12].

MSCs can be identified by their ability to adhere to plastic as well as to grow into colony forming unitsfibroblast (CFU-F) in vitro. However, these cells are heterogeneous with respect to their proliferation and differentiation capacity $[13,14]$. To define this population more precisely, surface markers that are enriched in MSCs, such as CD105 (SH2, endoglin), CD73 (SH3/SH4), SSEA-4, STRO-1 and CD271 (the low affinity nerve growth factor receptor), have been used

Abbreviations: MSCs - mesenchymal stem cells; BM - bone marrow; GvHD - graft-versus-host disease; CFU-F - colony forming units-fibroblast; BMMNCs - bone marrow mononuclear cells; ISCT - international society for cellular therapy; MNCs mononuclear cells 
[15-17]. To date, only a few markers have been developed and proved suitable for the isolation of MSCs from primary tissues. Markers that meet established criteria for their positive selection include STRO-1, CD73, CD105 and CD271, whereas CD45 and CD235 (glycophorin A) are used for the negative selection of MSCs [6,18-22]. The International Society for Cellular Therapy (ISCT) proposed three minimal criteria for defining MSCs: 1) these cell population must be plastic-adherent when maintained in standard culture conditions using tissue culture flasks; 2) $\geq 95 \%$ of the MSCs population must express CD105, CD73 and CD90, as measured by flow cytometry and lack expression ( $\leq 52 \%$ positive) of CD $45, \mathrm{CD} 34, \mathrm{CD} 14$ or CD11b, CD79a or CD19 and HLA class II; and 3) MSCs must be able to differentiate to osteoblasts, adipocytes and chondroblasts under standard in vitro differentiating conditions $[23,24]$.

In this work, we have considered the use of 5 monoclonal antibodies that are recommended by the ISCT [23]: two positive markers of MSCs (CD90 and CD105) and three negative markers of MSCs (CD34, CD45 and CD79). We tested if appropriate expression of these established MSC markers correlates with CD271 expression alone. Our results indicate that CD271 expression is a specific marker for the identification of fresh bone marrow MSCs. Using CD271 as the sole marker for MSC identification would reduce reagent costs and may potentially accelerate the identification of MSCs for cellular therapy.

\section{Materials and methods}

Isolation of bone marrow mononuclear cells. Adult human mononuclear cells (MNCs) were isolated from the bone marrow (BM) of 25 healthy volunteer donors after informed consent was obtained. A total of 4 to $5 \mathrm{ml}$ of $\mathrm{BM}$ was obtained by aspiration from the posterior iliac crest under local anesthesia, according to standard institutional procedures. MNCs were isolated by density gradient centrifugation (LymphoprepTM, AXIS-SHIELD PoC AS, Norway). Briefly, 2 volumes of BM were added to 1 volume of Lymphoprep and centrifuged at $1500 \mathrm{rpm}$ for 30 minutes. MNCs were then resuspended before cytometry in $1 \mathrm{ml}$ of Dulbecco's Phosphate Buffered Saline (GIBCO, InvitrogenTM, USA).

Characterization of MSCs immunophenotype. For fluorescence-activated cell analysis, the following antibodies were used: CD45 PE-TR (Caltag Laboratories U.S.A.), CD34 PE-Cy5 (Caltag Laboratories U.S.A.), CD79 alpha/APC (Dako, Denmark), CD90 FITC (Dako, Denmark), CD105 PE (eBioscience, U.S.A.), and separately CD271 (LNGFR) FITC (Miltenyi Biotec, U.S.A.).

Flow cytometry. A total of $1 \times 10^{6}$ cells were resuspended in 50 $\mu 1$ of Dulbecco's Phosphate Buffered Saline (PBS) and incubated with $10 \mu \mathrm{l}$ of antibody for 30 minutes in the dark at room temperature. Cells were resuspended in $1 \mathrm{ml}$ of Dulbecco's PBS and analyzed immediately. The analysis was performed on a Dako Cytomation flow cytometer (CyAnADP, Dako Colorado, Inc. U.S.A.). Calibration of the instrument was performed before data acquisition using well-established protocols. The software program Summit (Dako Cytomation) was used for the acquisition of
50,000 total cells. Data analysis was performed using Summit v4.3 (Dako Cytomation).

Statistical analysis. Statistical analyses of the differences between the two groups of used markers were performed using a Pearson's correlation test.

\section{Results \\ Immunophenotype}

We hypothesized that CD271 can be used as the sole marker for MSCs derived from BM. To test this, we isolated the MNC fraction from BM, incubated these cells with established cellular markers for MSCs and compared them to cells isolated by CD271 expression alone. As recommended by the ISCT for identifying MSCs, we sorted cells for the presence of the markers CD90 and CD105 and the absence of the markers CD45, CD34 and CD79. We then tested if the isolated population of MSCs correlated to the population identified by CD271 expression (Fig. 1). We found that the average percentage of isolated MNCs from $\mathrm{BM}$ that were $\mathrm{CD} 90^{+}, \mathrm{CD} 105^{+}, \mathrm{CD}_{45}^{-}, \mathrm{CD} 34^{-}$and $\mathrm{CD}^{-} 9^{-}$was $0.54 \%$, and that the average percentage of the population that was $\mathrm{CD} 271^{+}$was $0.53 \%$ (Fig. 2). A two-tailed Pearson's correlation test of 25 replicated experiments indicated that the correlation between these populations was significant $(\mathrm{p}<0.05)$. These results strongly suggest that CD271 alone is sufficient to identify MSCs.

\section{Discussion}

MSCs are marrow-derived progenitor cells that have been extensively investigated and characterized by their ability to self-renew and differentiate into multiple lineages including osteoblasts, adipocytes, fibroblasts, and chondrocytes [6,7,12-14,19]. The isolation procedure for MSCs continues to be based on their plastic adherence properties [7,25-28]. In addition, the nomenclature used to distinguish between isolated cells types is lacking. In an attempt to standardize nomenclature, the ISCT proposed in a position paper that cultured MSCs should be designated "multipotential mesenchymal stromal cells" whereas the term "mesenchymal stem cells" should be reserved for cells from primary tissues that can give rise to "colonyforming units-fibroblasts (CFU-F)" [6,13]. Later, the ISCT proposed minimal criteria to define human MSCs, as previously described [23].

Though MSCs are routinely isolated from MNCs primarily by plastic adherence, some authors have studied the CD271 marker as a specific indicator of the MSCs. Quirici et al. used CD271 to purify MSCs from adult BM and compared CD271 expression with CD45 expression and plastic adherence [19]. Jarocha et al. also considered CD271 as an indicator of MSCs and 

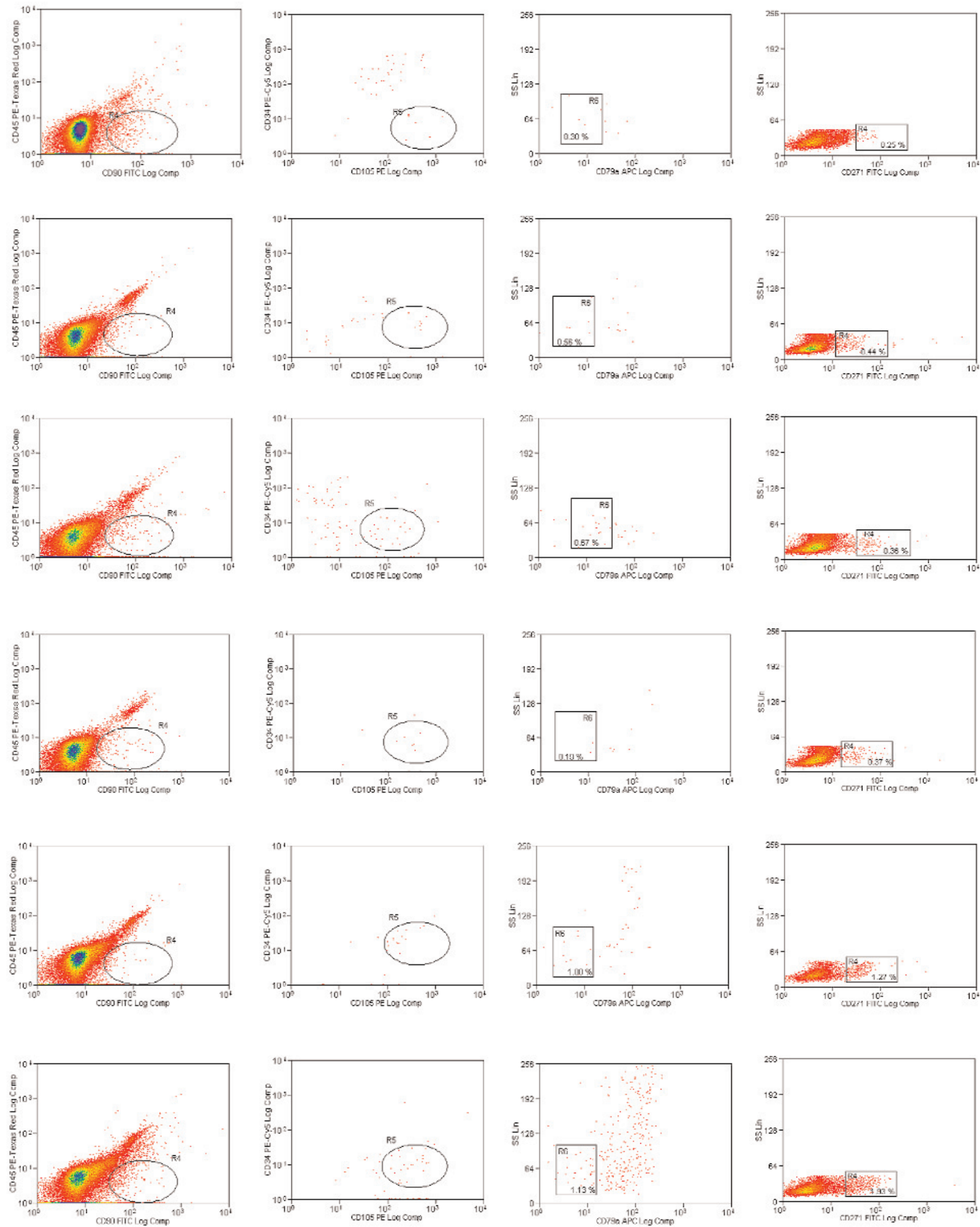

Fig. 1. MSC antigen expression. Analysis of mononuclear cells from bone marrow by flow cytometry. Six representative analysis out of 25 total experiments are shown comparing the percentage of $\mathrm{CD} 90^{+}, \mathrm{CD} 105^{+}, \mathrm{CD} 34^{-}, \mathrm{CD}^{-} 5^{-}$and $\mathrm{CD} 79^{-}$cells with the percentage of $\mathrm{CD} 271^{+}$cells. The result from a Pearson's correlation test was significant $(\mathrm{p}<0.05)$. 


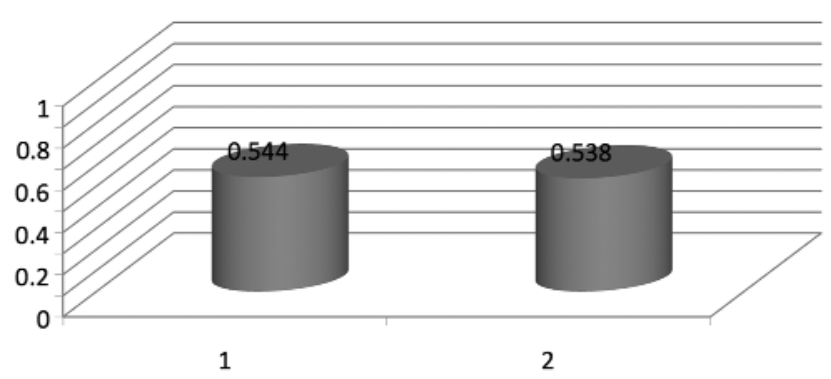

Fig. 2. Average percentage of cells. The graphic shows the average percentage of $\mathrm{CD}^{+} 0^{+}, \mathrm{CD} 105^{+}, \mathrm{CD} 45^{-}, \mathrm{CD} 34^{-}$and $\mathrm{CD}^{-} 9^{-}$cells (1) compared with average percentage average of $\mathrm{CD} 271^{+}$cells (2).

compared the differentiation capacity of $\mathrm{CD} 271^{+}$cells with cells expressing other markers such CD105 as well as with BM enriched for MSCs by RosetteSep Isolation Kit and non-purified bone marrow mononuclear cells (BMMNC). They concluded that MSCs can be defined by the following parameters: a) their efficacy to form CFU-F colonies; b) their expression of MSCs-associated antigens (CD105, CD166, CD44, CD73, CD45, CD34) and genes (e.g. RUNX2, PPAR 2 , osteocalcin, N-cadherin); and c) their adipocyto/osteogenic potential in vitro [7]. In a separate study, Battula et al. used CD271 expression together with the property of plastic adherence (CFUF) to isolate of a new subset of MSCs [14]. Poloni et al. used CD271 to isolate MSCs from BM and showed it was feasible to expand MSCs in medium supplemented with $10 \%$ pooled allogeneic human serum without loss of multipotent differentiation capacity or karyotype alterations [29]. Until recently, CD271 has been described as the most selective marker for the characterization and purification of human Bone Marrow-Mesenchymal Stem Cell [6]. Likewise, Zhou et al. proposed to detect the expression of CD271, CD133 and CD34 and to analyze the correlation of CD271 with CD133 as well as CD133 with CD34 expression by flow cytometry with three color fluorescence labeling. They concluded that $\mathrm{CD} 271^{+}$cells are different from $\mathrm{CD}_{133^{+}}$and $\mathrm{CD} 34^{+}$cells, which suggests that CD271 might have an important role in evaluating MSCs and therefore guiding their future clinical application [30].

\section{Conclusions}

The objective of this study was to analyze the correlation of CD271 expression with the positive expression of CD90, CD105 markers as well as the negative expression of CD34, CD45 and CD79 markers on cells from fresh human BM. Our goal was to determine the feasibility of using CD271 expression as a marker for MSCs and to demonstrate that the exclusive use of this marker is sufficient to identify MSCs from fresh samples of BM cells. BMCs and MNCs were detected by flow cytometry. We found in 25 samples of fresh BM that the correlation between the previously established markers and CD271 expression as assessed by a Pearson's test was highly significant $(\mathrm{p}<0.05)$. These results agree with the works of Battula, Buhring, Jarocha, and Poloni which also used the CD271 marker as a reference to isolate and to identify MSCs. Because some cellular therapy treatments start with fresh BM, the ability to quantify MSCs quickly is essential. Furthermore, the use of a single marker to identify MSCs allows for a convenient, stream-lined protocol without the extensive analysis required by using a series of defined markers and without requiring culture in plastic dishes. In addition, we have demonstrated that CD271 expression alone can substitute for established phenotypic markers in isolating MSCs.

Competing interests: The authors declare that they have no competing interests. Authors' contributions: All authors participated in the drafting of the manuscript. EF and $\mathrm{AO}$ edited the manuscript and $\mathrm{OG}$ and $\mathrm{AC}$ read and approved the final manuscript.

\section{References}

[ 1] Keating A. Mesenchymal stromal cells. Curr Opin Hematol. 2006;13:419-425.

[2] Fibbe W. Mesenchymal stem cells. A potential source for skeletal repair. Ann Rheum Dis. 2002;61(Suppl II):ii29-ii31.

[3] Deans RJ, Moseley AB. Mesenchymal stem cells: biology and potential clinical uses. Exp Hematol. 2000;28:875-884.

[4] Erices A, Conget P, Minguell JJ. Mesenchymal progenitor cells in human umbilical cord blood. $\mathrm{Br} J$ Haematol. 2000;109:235-242.

[5] Zhao LR,. Duan WM, Reyes M, et al. Human bone marrow stem cells exhibit neural phenotypes and ameliorate neurological deficits after grafting into the ischemic brain of rats. Exp Neurol. 2002;174:11-20.

[6] Bühring H-J, Battula VL, Treml S, Schewe B, Kanz L, Vogel W. Novel Markers for the Prospective Isolation of Human MSC. Ann NY Acad Sci. 2007;1106:262-271.

[7] Jarocha D, Lukasiewicz E, Majka M. Adventage of mesenchymal stem cells (MSC) expansión directly from purified bone marrow CD105+ and CD271+ cells. Folia Histochem Cytobiol. 2008;46(3):307-314.

[ 8] Smits AM, Van Vliet P, Hassink RJ, Goumans MJ, Doevendans PA. The role of stem cells in cardiac regeneration. J Cell Mol Med. 2005;9:25-36.

[9] Horwitz EM, Prockop DJ, Fitzpatrick LA, et al. Transplantability and therapeutic effects of bone marrow-derived mesenchymal cells in children with osteogenesis imperfecta. Nat Med. 1999;5:309-33.

[10] Bobis S, Jarocha D, Majka M. Mesenchymal stem cells: characteristics and clinical applications. Folia Histochem Cytobiol. 2006;44:215-230.

[11] Psaltis P J, Zannettino A, Worthley SG, Gronthos S: Mesenchymal Stromal Cells - Potential for Cardiovascular Repair. Stem Cells Express. published online July 3, 2008; doi:10.1634/stemcells.2008-0428.

[12] Uccelli A, Moretta L, Pistoia V. Mesenchymal stem cells in health and disease. Nat Rev Immunol. 2008;8:726-736. 
[13] Horwitz EM, Le BK, Dominici M, Mueller I, Slaper-Cortenbach I, Marini FC, et al. Clarification of the nomenclature for MSC: the International Society for Cellular Therapy position statement. Cytotherapy. 2005;7:393-395.

[14] Battula VL, Treml S, Bareiss PM, Gieseke F, Roelofs H, de Zwart P, Müller I, Schewe B, Skutella T, Fibbe WE, Kanz L, Bühring H-J: Isolation of functionally distinct mesenchymal stem cells subsets using antibodies against CD56, CD271, and mesenchymal stem cell antigen-1. Haematologica. 2009;94:173-184.

[15] Battula VL, Bareiss PM, Treml S, Conrad S, Albert I, Hojak $\mathrm{S}$, et al. Human placenta and bone marrow derived MSC cultured in serum-free, b-FGF-containing medium express cell surface frizzled-9 and SSEA-4 and give rise to multilineage differentiation. Differentiation. 2007;75:279-91.

[16] Jones EA, Kinsey SE, English A, et al. Isolation and characterization of bone marrow multipotential mesenchymal progenitor cells. Arthritis Rheum. 2002;46:3349-3360.

[17] Meirelles L da S, Chagastelles P C, Nardi NB. Mesenchymal stem cells reside in virtually all postnatal organs and tissues. J Cell Sci. 2006;119:2204-2213.

[18] Sabatini F, Petecchia L, Tavian M, et al. Human bronchial fibroblasts exhibit a mesenchymal stem cell phenotype and multilineage differentiating potentialities. Lab Invest. 2005;85:962-971.

[19] Quirici N, Soligo D, Bossolasco P, et al. Isolation of bone marrow mesenchymal stem cells by anti-nerve growth factor receptor antibodies. Exp Hematol. 2002;30:783-791.

[20] Jiang Y, Jahagirdar B, Reinhardt R, et al. Pluripotency of mesenchymal stem cells derived from adult marrow. Nature. 2002;418:41-49.

[21] Verfaillie, C. Stem cell plasticity. Hematology. 2005;10(Suppl 1):293-296.

[22] Verfaillie, CM. Multipotent adult progenitor cells: an update. Novartis Found Symp. 2005;265:55-61.

[23] Dominici M, Le Blanc K, Mueller I, Slaper-Cortenbach I, Marini FC, Krause DS, Deans RJ, Keating A, Prockop DJ,
Horwitz EM. Minimal criteria for defining multipotent mesenchymal stromal cells. The International Society for Cellular Therapy position statement. Cytotherapy. 2006;8(4):315-317.

[24] Horwitz EM. COMMENTARY: Mesenchymal stromal cells moving forward. Cytotherapy. 2008;10(1):5-6.

[25] Angelopoulou M, Novelli E, Grove JE et al. Cotransplantation of human mesenchymal stem cells enhances human myelopoiesis and megakaryocytopoiesis in NOD/SCID mice. Exp Hematol. 2003;31:413-420.

[26] Vidal MA, Kilroy GE, Johnson JR, Lopez MJ, Moore RM, Gimble JM: Cell growth characteristics and differentiation frequency of adherent equine bone marrow-derived mesenchymal stromal cells: adipogenic and osteogenic capacity. Vet Surg. 2006;35:601-610.

[27] Maccario R, Podesta M, Moretta A, et al. Interaction of human mesenchymal stem cells with cells involved in alloantigen-specific immune response favors the differentiation of CD4+ T-cell subsets expressing a regulatory/suppressive phenotype. Haematologica. 2005;90:516-525.

[28] Tolar J, Nauta AJ, Osborn MJ et al. Sarcoma derived from cultured mesenchymal stem cells. Stem Cells. 2007;25:371379.

[29] Poloni A, Maurizi G, Rosini V, Mondini E, Mancini S, Discepoli G, Biasio S, Battaglini G, Felicetti S, Berardinelli E, Serrani F, Leoni P. Selection of CD271+ cells and human $\mathrm{AB}$ serum allows a large expansion of mesenchymal stromal cells from human bone marrow. Cytotherapy. 2009;11(2): $153-162$

[30] Zhou J, Zhu B, DU HY, Sun TS, Zhang CH, Yang LG: Detection and analysis of CD271, CD133 and CD34 expressions in bone marrow cells by flow cytometry with three color fluorescence labeling [abstract]. Zhongguo Shi Yan Xue Ye Xue Za Zhi. 2009;17(1):133-6.

Submitted: 7 May, 2010 Accepted after reviews: 5 August, 2010 